\title{
Excited state intramolecular charge transfer reaction of 4-(morpholenyl) benzonitrile in solution: Effects of hetero atom in the donor moiety
}

\author{
TUHIN PRADHAN, HARUN AL RASID GAZI and RANJIT BISWAS* \\ Department of Chemical, Biological and Macromolecular Sciences, \\ S.N. Bose National Centre for Basic Sciences, J.D. Block, Sector III, Salt Lake, Kolkata 700098 \\ e-mail: ranjit@bose.res.in
}

MS received 14 January 2010; revised 16 June 2010; accepted 18 June 2010

\begin{abstract}
An intramolecular charge transfer (ICT) molecule with an extra hetero atom in its donor moiety has been synthesized in order to investigate how ICT reaction is affected by hetero atom replacement. Photo-physical and photo-dynamical properties of this molecule, 4-(morpholenyl)benzonitrile (M6C), have been studied in 20 different solvents. The correlation between the reaction driving force $\left(-\Delta G_{r}\right)$ and activation barrier $\left(\Delta G^{\#}\right)$ has been explored in order to understand the solvent effects (static and dynamic) on the photo-excited ICT reaction in this molecule. A Kramer's model analysis of the experimentally observed reaction rate constants indicates a solvent-averaged activation barrier of $\sim 4 k_{B} T$ in the absence of solvent dynamical control. The reaction in $\mathrm{M} 6 \mathrm{C}$ is therefore not a barrier-less reaction but close to the limit where conventional kinetics might break down.
\end{abstract}

Keywords. ICT reaction; solvent effects; hetero atom replacement.

\section{Introduction}

Excited state intramolecular charge transfer (ICT) reactions in substituted aminobenzonitrile derivatives have been an area of intense research for quite some time now. ${ }^{1-5}$ While potential applications of ICT molecules in designing fluorescent dyes, ${ }^{6-7}$ electron transfer photochemistry, ${ }^{8}$ solar collectors, ${ }^{7}$ $\mathrm{pH}$ or ion indicators ${ }^{9,10}$ have driven much of the interest in industry, quest for fully understand the basic scientific aspects of ICT reactions in various media continues to attract attention of physical chemists. Photo-induced charge transfer is an important process in biology as light-induced charge separation plays key roles in photosynthesis and vision. Since a small barrier of a few $k_{B} T$ is sometimes associated with the ICT reaction in many molecules, interpretation of static and dynamic solvent control of reaction in these molecules becomes more complex. A twostate model is sometimes used to describe the excited state ICT reaction which, in the absence of solvation dynamics within the observation time, predicts a bi-exponential kinetics. ${ }^{5,11-13}$ A deviation from the bi-exponential kinetics then signals the break-down of the conventional high-barrier kinetics.

*For correspondence
The molecule that we are considering in this work is 4-(morpholenyl)benzonitrile (M6C) which contains an oxygen atom in a position para to the nitrogen atom of the donor moiety (see scheme 1). Typical to
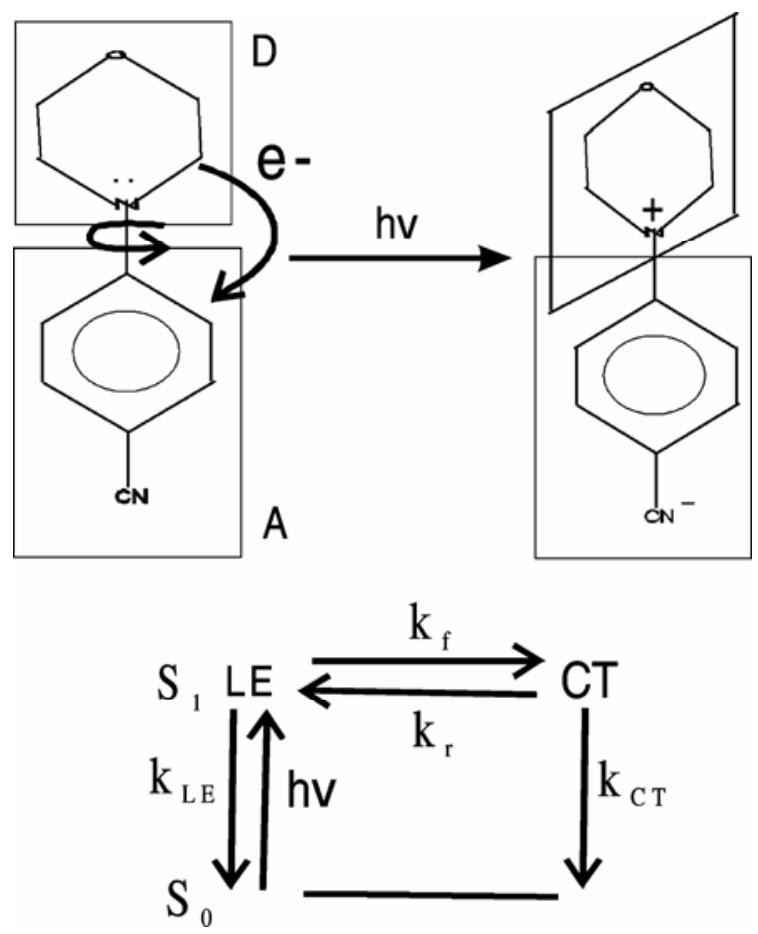

Scheme 1. 
many DMABN derivatives, this molecule also show two fluorescence emission bands in polar solvents and only one band in non-polar solvents. The appearance of two emission bands in polar solvents is a reflection of intramolecular charge transfer reaction in the excited state and several models have been proposed to explain the new emission in polar environments. ${ }^{1}$ The new emission in polar molecules are believed to occur from an electronic state of charge transfer (CT) character, whereas the normal fluorescence is from a locally excited (LE) character with charge distribution similar to that in the ground state. Upon photoexcitation, a substantial amount of charge is transferred from the amino group to benzonitrile moiety forming the CT state. Consequently, the more polar CT state is further stabilized by polar solvent via enhanced dipole-dipole interaction between dipolar solvent molecules and photoexcited solute.

We will use the twisted intramolecular charge transfer (TICT) model to explain the photo-induced charge transfer reaction in the M6C. Eventhough other models are also used to explain the CT emission in hindered DMABN derivatives, TICT model has been found to be suitable for unhindered molecules like the one which is being considered in this work. According to the twisted intramolecular charge transfer (TICT) model, the charge transfer process occurs with the simultaneous twisting of the bond connecting the amino group and the benzene ring. ${ }^{1-5}$ Further evidence for the TICT mechanism comes from the experimentally observed solvent dynamical control of the LE $\rightarrow$ CT conversion reaction rate. ${ }^{12-15}$ The agreement between theory and experiments found in our earlier studies in electrolyte solutions $^{12,13}$ also provides support to the TICT mechanism for the excited state intramolecular charge transfer reaction. As shown in scheme 1, photo-excitation promotes $\mathrm{M} 6 \mathrm{C}$ to the locally excited (LE) state in the first excited electronic surface $\left(S_{1}\right)$. The photo prepared LE state then either undergoes intramolecular charge transfer with the forward reaction rate constant $k_{f}$ or comes back to the ground state $\left(S_{0}\right)$ with an average (radiative + nonradiative) rate constant $\left(k_{\mathrm{LE}}\right)$. Likewise, the charge transferred (CT) state can go back to the LE state with a rate constant of $k_{r}$ or populate the ground state via the average (radiative and non-radiative) rate constant $k_{\text {Ст }}$. As already discussed ${ }^{5}$ such a two state model with time-independent rate constants is expected to generate bi-exponential emission decays for M6C in polar solvents, provided solvent dynamics is not seen during the time scale of the interconversion reaction.

The main results of the paper are as follows Quantum yield, radiative and non-radiative rates, transition moments and changes in reaction free energy (reaction driving force) for M6C have been determined in $\sim 20$ different solvents and compared with a closely related molecule, 4-(1-piperidinyl)benzonitrile $\mathrm{P} 6 \mathrm{C}$. These solvents provide a good spread of solvent field factor as the static dielectric constant $\left(\varepsilon_{0}\right)$ ranges from $\sim 2$ to 110 . Bi-exponential decay kinetics has been found for M6C in all the polar solvents studied and therefore the LE $\rightarrow$ CT interconversion reaction in this molecule conforms to the two-state reversible reaction mechanism. ${ }^{5}$ Correlations between the solvent polarity and changes in reaction free energy, and reaction rate constant are attempted to explore the solvent effects on the ICT reaction in this molecule. Average activation energy has been estimated by using Kramer's type model for the barrier crossing and a barrier of $\sim 4 k_{B} T$ is found in the absence of dynamic solvent effects on the ICT reaction. Note that the ICT molecule under study (M6C) has been used in of our earlier study ${ }^{16(a)}$ to characterize the encapsulated polar solvent pools inside reverse micelles. In that study, the average rate-constant of TICT reaction in this molecule has been used as a probe to acquire information about the static and dynamic medium effects on chemical reaction $^{16(b)}$ in confined aqueous environments. A comparatively larger solubility of M6C in water has been particularly helpful in that study. ${ }^{16(a)}$ However, the photophysics and photodynamics of M6C have not been studied before and the present work provides a thorough study of these aspects of this ICT molecule in bulk solvents at ambient condition

The organization of the rest of the article is as follows. Experimental details are discussed in the next section. Section 3 contains the experimental results, analysis of which are based on equations derived in ref. 5 and discussion on photophysics and photodynamics of M6C molecule in various solvents. Finally, the concluding remarks are given in section 4 .

\section{Experimental}

4-(1-morpholenyl) benzonitrile (M6C) was synthesized by following the protocol given in the literature. ${ }^{17}$ Steady state absorption spectra were recorded by using a spectrophotometer (Shimadzu, UV-2450). 
Steady state emission spectra were recorded by using a fluorimeter (SPEX fluoromax-3, Jobin-Yvon, Horiba) after adjusting the absorbance of the sample to $\sim 0 \cdot 1$. Measurements were made by taking the solutions in an optically transparent, quartz cuvette with $1 \mathrm{~cm}$ optical path length. For a given sample, the peak wavelength $(\lambda)$ of the absorption spectrum was used as excitation wavelength for the corresponding emission scan. The fluorescence spectra were properly corrected before extracting relevant quantities from them. Samples bubbled with dry nitrogen gas show no effects on the overall appearance of the spectra for M6C in solutions and also on its decay kinetics.

Average peak frequencies of the absorption and emission spectra were obtained from various measures of the frequencies as before. The area under the LE and CT emission bands were determined by shifting and broadening a reference emission spectrum of $\mathrm{M} 6 \mathrm{C}$ in a non-polar solvent, keeping constant the total area under the emission spectrum in a given polar solvent. The error associated with the peak frequency determination is typically $\pm 250 \mathrm{~cm}^{-1}$ and that with the band area is $\sim 10 \%$ (of the reported value), unless otherwise mentioned.

Time-resolved fluorescence emission intensity decay for M6C in these solvents were collected by using a time-correlated single photon counting (TCSPC) instrument based on a picosecond Ti: sapphire laser with excitation light wavelength at $300 \mathrm{~nm}$ (third harmonic). The emission fluorescence was collected at magic angle at both LE and CT peak positions (of steady-state spectrum) with an emission band-pass of $8 \mathrm{~nm}$. The effective resolution (full width at half maximum, fwhm) of the instrument response function (IRF) was $\sim 100$ ps. Subsequently, decays were deconvoluted from IRF and fitted to multiexponential function using an iterative reconvolution algorithm. ${ }^{5}$ Such fitting enables one to capture decay kinetics with time constant as fast as $\sim 20$ ps with reasonable accuracy. For a few samples, emission decays collected at a few wavelengths near the LE or CT peak positions were analysed and the analysed data were found to vary within a small uncertainty. Note that emission intensity decays for M6C in non-polar solvents were found to be singleexponential collected at any wavelength across its steady state emission band. These decays fit with one large time constant which is different from the small time constant obtained from the bi-exponential decay in polar solvents. All the measurements reported here were performed at $T(\mathrm{~K})=298 \pm 0 \cdot 1$.

\section{Results and discussion}

Figure 1 displays absorption and emission spectra of M6C in heptane $\left(\varepsilon_{0} \sim 2\right)$, ethyl acetate $\left(\varepsilon_{0} \sim 6\right)$ and acetonitrile $\left(\varepsilon_{0} \sim 36\right)$. As expected, both the absorption and emission spectra show red-shift as the solvent becomes more polar. In addition, both the LE and CT emission bands appear as the non-polar heptane is replaced by a moderately polar solvent, ethyl acetate. When the solvent's polarity becomes as large as that of acetonitrile, CT emission absolutely dominates the spectrum. Similar polarity-induced domination of CT population has been observed earlier ${ }^{5}$ with a closely related molecule, P6C. However, both absorption and emission spectra in a given solvent of these two molecules (M6C and P6C) differ appreciably, eventhough both these molecules possess very similar six-membered rings as donor groups. A representative of this sort is presented in

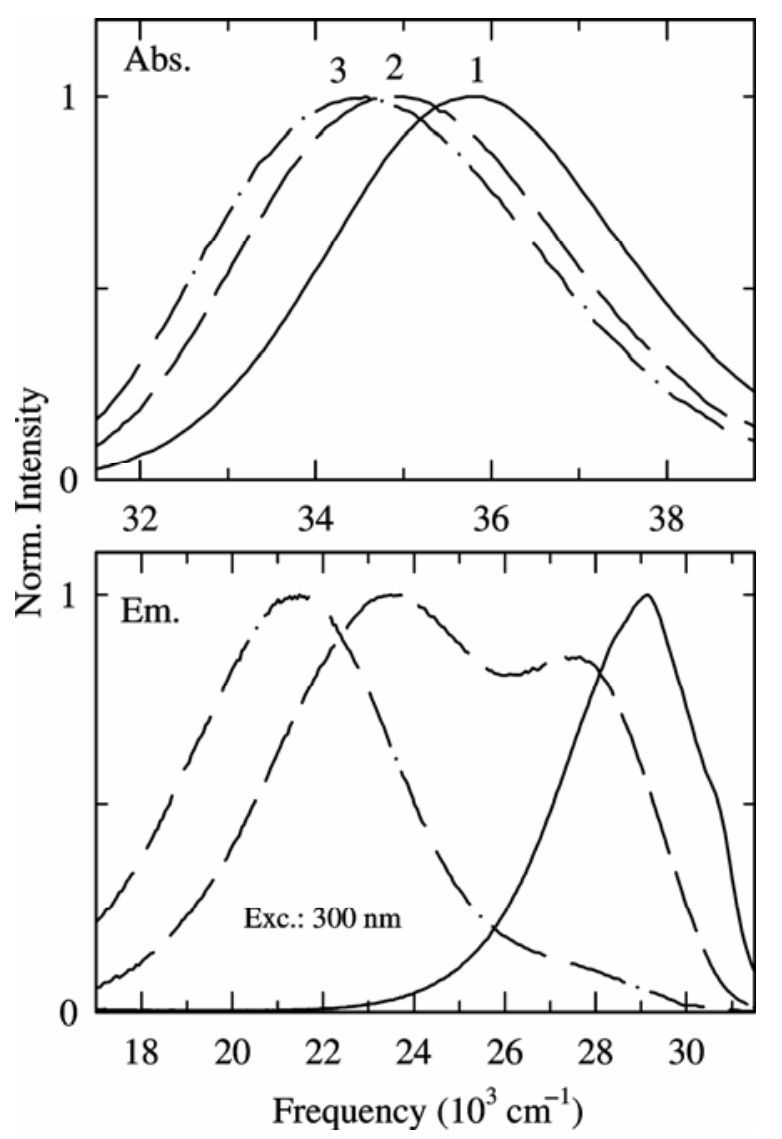

Figure 1. Absorption and emission spectra of 4-(1morpholenyl) benzonitrile (M6C) in solvents of differing polarities: heptane (solid line, 1), ethyl acetate (long dash, 2), acetonitrile (dash-dot, 3 Upper panel represents the absorption spectra and lower panel the emission spectra. 
figure 2 where absorption and emission spectra of these two molecules in ethyl acetate are shown. It is evident from this figure that both the absorption and CT emission bands of $\mathrm{M} 6 \mathrm{C}$ is $\sim 700 \mathrm{~cm}^{-1}$ blueshifted than those for $\mathrm{P} 6 \mathrm{C}$ in ethyl acetate. This indicates that both the ground and excited states of $\mathrm{M} 6 \mathrm{C}$ are less polar than in $\mathrm{P} 6 \mathrm{C}$, signalling relatively more contribution of the less polar $L_{b}$ state in the transition moments of M6C. ${ }^{5}$

Characteristics of the absorption and emission spectra of M6C were determined in 20 different solvents that provide a good spread of the dielectric reaction field factor,

$$
d_{c}\left(\varepsilon_{0}\right)=\frac{\varepsilon_{0}-1}{2(1-c) \varepsilon_{0}+(1+2 c)},
$$

where $c$, ranging between 0 and 1 , effectively accounts for solute polarizability effects. We have used $c=0.25$ since this produces the best correlation. ${ }^{5}$

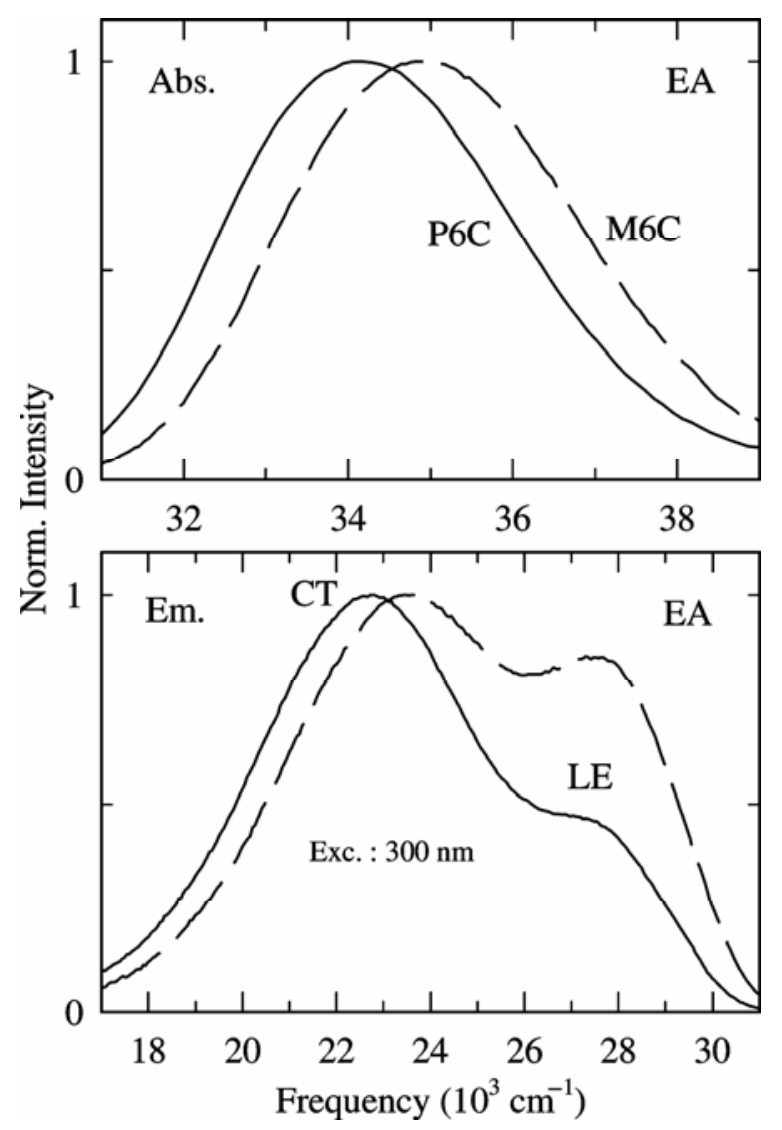

Figure 2. A comparison between the absorption (upper panel) and emission (lower panel) spectra of 4-(1morpholenyl) benzonitrile (M6C) and 4-(1-piperidinyl) benzonitrile (P6C) in ethyl acetate. Note the relative blue shift in the spectra of M6C.
Table 1 lists these solvents and summarizes the steady state spectral data. Since one of our goals is to understand the solvent effects (both static and dynamic) on the ICT reaction in $\mathrm{M} 6 \mathrm{C}$, we explored correlations of various spectral quantities with the reaction field factor described above. One of such correlations is presented in figure 3 . The first two panels of this figure are essentially the depiction of what has already been observed in figure 1 for a few solvents. Data in protic polar solvents are shown by separate symbols (triangles) in order to find out if the solute-solvent specific (H-bonding) interaction plays any role. It is interesting to note that the area ratio, $\alpha_{C T} / \alpha_{L E}$ in methanol is $\sim 2.5$ times larger than

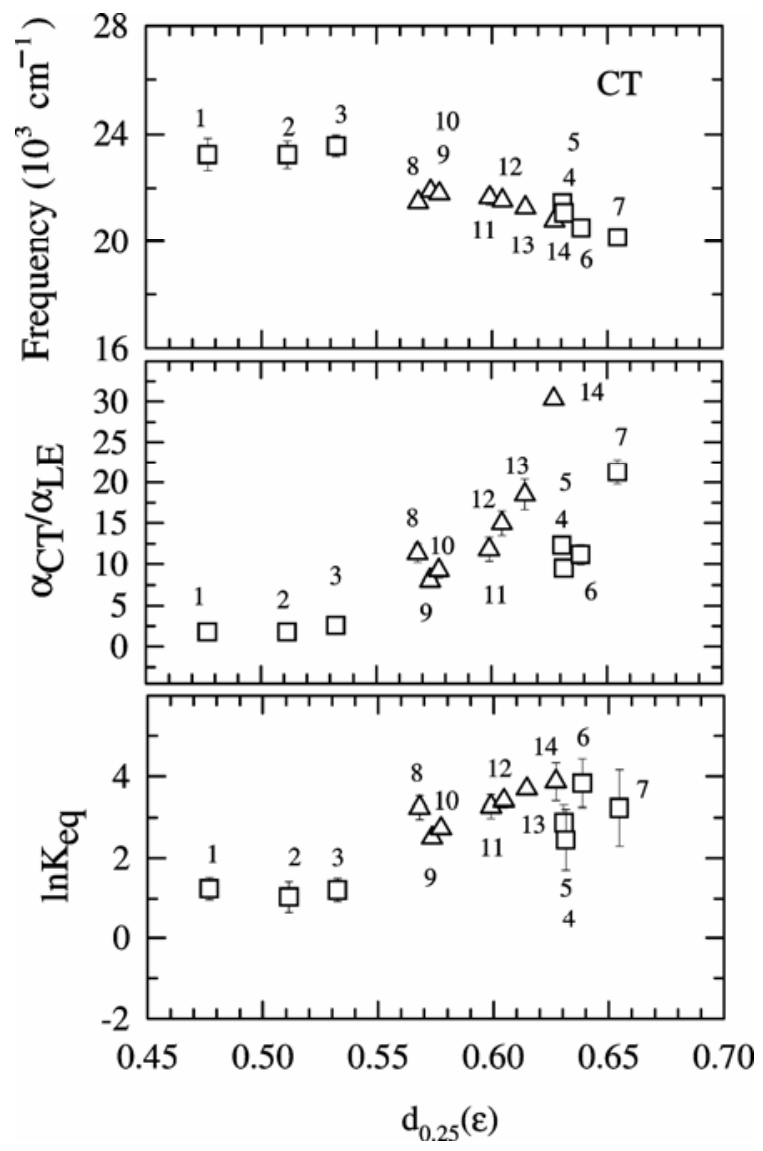

Figure 3. Correlations of peak frequency associated with $\mathrm{CT}$ band, the ratio $\left(\alpha_{C T} / \alpha_{L E}\right)$ between areas under the $C T$ and $L E$ bands, logarithm of equilibrium constant $\left(K_{e q}\right)$ with solvent dielectric factor, $d_{0.25}\left(\varepsilon_{0}\right)$. Data for protic polar solvents are represented by triangles and those for aprotic solvents by squares. Solvents are marked with numbers, which are assigned as follows: 1 (EA), 2 (THF), 3 (DCM), 4 (acetonitrile), 5 (DMF), 6 (DMSO), 7 (formamide), 8 (TBA), 9 (hexanol), 10 (pentanol), 11 (2-methyl-1-propanol), 12 (propanol), 13 (ethanol), 14 (methanol). Large error bars associated measurements in those solvents where one of the bands had less than $10 \%$ of the total area under the whole emission band. 
Table 1. Steady state spectral properties of M6C in different solvents.

\begin{tabular}{|c|c|c|c|c|c|c|c|c|c|c|c|}
\hline Solvent & $\varepsilon_{0}$ & $n_{D}$ & $v_{\mathrm{abs}}$ & $v_{L E}$ & $v_{C T}$ & $\Gamma_{a b s}$ & $\Gamma_{L E}$ & $\Gamma_{C T}$ & $\alpha_{C T} / \alpha_{L E}$ & $K_{e q}$ & $-\Delta G_{r}$ \\
\hline $\mathrm{PFH}$ & 1.76 & $1 \cdot 252$ & $36 \cdot 56$ & $29 \cdot 04$ & - & $3 \cdot 70$ & $3 \cdot 61$ & - & - & & \\
\hline Heptane & 1.92 & $1 \cdot 388$ & $35 \cdot 85$ & $28 \cdot 83$ & - & 3.98 & $3 \cdot 67$ & - & - & & \\
\hline Hexane & $2 \cdot 00$ & $1 \cdot 375$ & $35 \cdot 92$ & - & - & $3 \cdot 97$ & - & - & - & & \\
\hline Cyclohexane & $2 \cdot 02$ & 1.424 & $35 \cdot 74$ & $28 \cdot 66$ & - & $3 \cdot 96$ & $3 \cdot 63$ & - & - & & \\
\hline Diethyl ether & $4 \cdot 20$ & $1 \cdot 350$ & $35 \cdot 41$ & $27 \cdot 66$ & - & $4 \cdot 16$ & $3 \cdot 97$ & - & - & $0 \cdot 13$ & $-5 \cdot 14$ \\
\hline Ethyl acetate & $6 \cdot 02$ & $1 \cdot 370$ & $35 \cdot 04$ & 27.78 & $23 \cdot 24$ & $4 \cdot 30$ & $1 \cdot 18$ & $5 \cdot 80$ & 1.78 & $3 \cdot 48$ & 3.09 \\
\hline $\mathrm{THF}$ & $7 \cdot 58$ & 1.405 & $34 \cdot 66$ & $27 \cdot 78$ & $23 \cdot 24$ & $4 \cdot 02$ & $1 \cdot 18$ & $5 \cdot 80$ & 1.78 & $2 \cdot 83$ & $2 \cdot 57$ \\
\hline DCM & $8 \cdot 93$ & 1.421 & 34.66 & 27.79 & $23 \cdot 58$ & $4 \cdot 31$ & $0 \cdot 94$ & $3 \cdot 83$ & $2 \cdot 62$ & $3 \cdot 36$ & $3 \cdot 00$ \\
\hline TBA & $12 \cdot 5$ & $1 \cdot 387$ & $34 \cdot 88$ & $27 \cdot 38$ & $21 \cdot 48$ & $4 \cdot 55$ & $2 \cdot 09$ & $4 \cdot 32$ & $11 \cdot 32$ & $25 \cdot 51$ & $8 \cdot 03$ \\
\hline Hexanol & $13 \cdot 3$ & 1.415 & 34.79 & $27 \cdot 61$ & 21.90 & $4 \cdot 54$ & 1.60 & $4 \cdot 35$ & 8.05 & $12 \cdot 19$ & $6 \cdot 2$ \\
\hline Pentanol & $13 \cdot 9$ & 1.407 & $34 \cdot 77$ & $27 \cdot 55$ & $21 \cdot 80$ & $4 \cdot 55$ & 1.75 & $4 \cdot 31$ & $9 \cdot 24$ & $15 \cdot 31$ & $6 \cdot 76$ \\
\hline IBA & $18 \cdot 7$ & $1 \cdot 396$ & 34.77 & $27 \cdot 39$ & 21.66 & $4 \cdot 54$ & 1.84 & $4 \cdot 27$ & 11.80 & $26 \cdot 15$ & $8 \cdot 09$ \\
\hline Propanol & $20 \cdot 45$ & $1 \cdot 384$ & $34 \cdot 76$ & $27 \cdot 56$ & $21 \cdot 55$ & $4 \cdot 54$ & 1.66 & $4 \cdot 39$ & 14.97 & $30 \cdot 32$ & $8 \cdot 46$ \\
\hline Ethanol & $24 \cdot 55$ & $1 \cdot 359$ & $34 \cdot 80$ & $27 \cdot 45$ & $21 \cdot 28$ & $4 \cdot 56$ & 1.69 & $4 \cdot 37$ & $18 \cdot 49$ & $40 \cdot 25$ & $9 \cdot 16$ \\
\hline Methanol & 32.66 & $1 \cdot 327$ & 34.78 & $27 \cdot 31$ & $20 \cdot 79$ & $4 \cdot 6$ & 1.73 & $4 \cdot 39$ & $30 \cdot 35$ & $48 \cdot 21$ & $9 \cdot 61$ \\
\hline Acetonitrile & 35.94 & $1 \cdot 342$ & $34 \cdot 71$ & $27 \cdot 04$ & 21.46 & $4 \cdot 36$ & 1.96 & $3 \cdot 92$ & $12 \cdot 30$ & $17 \cdot 76$ & $7 \cdot 13$ \\
\hline DMF & $36 \cdot 71$ & $1 \cdot 428$ & $34 \cdot 49$ & $26 \cdot 45$ & $21 \cdot 08$ & - & $2 \cdot 82$ & $3 \cdot 67$ & $9 \cdot 48$ & $11 \cdot 62$ & $6 \cdot 08$ \\
\hline DMSO & $46 \cdot 45$ & 1.478 & $34 \cdot 09$ & $25 \cdot 85$ & $20 \cdot 53$ & $4 \cdot 38$ & $3 \cdot 36$ & $3 \cdot 61$ & $11 \cdot 15$ & $46 \cdot 34$ & $9 \cdot 51$ \\
\hline Formamide & 111.00 & 1.447 & $34 \cdot 18$ & $25 \cdot 47$ & $20 \cdot 16$ & $4 \cdot 57$ & $2 \cdot 5$ & $3 \cdot 93$ & $21 \cdot 27$ & $25 \cdot 22$ & $8 \cdot 00$ \\
\hline
\end{tabular}

Avg. frequency $(v)$ and full width at half maxima $(\Gamma)$ are expressed in $10^{3} \mathrm{~cm}^{-1} .-\Delta G_{r}$ are in kJ mol ${ }^{-1}$ units. PFH: perfluorohexane, DCM: Dichloromethane, THF: Tetrahydrofuran, TBA: Tertiary butyl alcohol, IBA: Isobutyl alcohol, DMF: Dimethyl formamide, DMSO: Dimethyl sulfoxide.

that in acetonitrile eventhough the latter is somewhat more polar than the former. Earlier studies with $\mathrm{P} 6 \mathrm{C}$ also reported similar relative enhancement in methanol. ${ }^{5}$ Whether these results indicate $\mathrm{H}$ bonding interaction between $\mathrm{M} 6 \mathrm{C}$ and polar protic solvents is not certain but it is clear in this figure that area ratio is uniformly larger in polar protic solvents than that in the polar aprotic ones. The inverse correlation between the CT frequency and the area ratio, $\alpha_{C T} / \alpha_{L E}$, for M6C in pure solvents suggest that the dielectric filed factor is responsible for both the red shift in the CT emission spectrum and enhancement of its population.

Since the area ratio is increasing linearly with the solvent dielectric field factor, the equilibrium constant for the LE $\rightarrow$ CT conversion reaction in M6C is also expected to increase in the similar manner. The last panel of figure 3 shows the expected behaviour. Note that the equilibrium constant shown in this panel is a mean of the equilibrium constants determined from the steady state and time resolved studies. The steady state equilibrium constant is obtained from the area ratio, ${ }^{5}$

$$
K_{e q}^{\alpha} \approx \frac{\alpha_{C T} v_{L E}^{3}}{\alpha_{L E} v_{C T}^{3}}
$$

where the frequency factor takes care of the difference in radiative rates between the LE and CT states. Equilibrium constant can be estimated from time resolved studies by simply taking the ratio between the decay components, $K_{e q}^{a}=a_{r x n} / a_{d e c}$. Note $a_{r x n}$ is the amplitude of the component associated with the short (reaction) time constant and $a_{d e c}$ with the long (population decay) time constant. It is clear from this panel that the average equilibrium constant $\left(K_{e q}\right)$ increases by a factor of $\sim 50$ on changing the solvent from diethyl ether $\left(\varepsilon_{0} \sim 4\right)$ to acetonitrile $\left(\varepsilon_{0} \sim 36\right)$. The strong correlations depicted in these three panels therefore suggest that the ICT reaction in $\mathrm{M} 6 \mathrm{C}$ involves a sizeable barrier and the solvent polarity modifies it significantly. However, these correlations cannot indicate the extent of solvent dynamical control of the same reaction in these solvents. Correlation between the reaction rate and changes in reaction free energy $\left(-\Delta G_{r}\right)$ found in earlier studies ${ }^{5}$ have indicated a rather limited role of solvent dynamical modes in modifying the reaction rate in these unhindered DMABN derivatives.

Before we present experimental results on the solvent dependence of the reaction rate constant and its correlation with solvent dielectric properties, let us investigate the solvent dependence of other solute properties, such as, quantum yield, radiative and non-radiative rates, and transition moments (abosorption and emission). We have measured these quantities for M6C in many of these solvents and the data are summarized in table 2 . The relations 
Table 2. Quantum yield, radiative, non-radiative rates of M6C in different solvents.

\begin{tabular}{|c|c|c|c|c|c|}
\hline Solvent & $\Phi_{\text {net }}$ & $\Phi_{\mathrm{LE}}$ & $\Phi_{\mathrm{CT}}$ & $k_{L E}^{r a \mathrm{~d}}\left(10^{7} \mathrm{~s}^{-1}\right)$ & $k_{L E}^{n r}\left(10^{9} \mathrm{~s}^{-1}\right)$ \\
\hline PFH & 0.033 & 0.033 & - & $2 \cdot 53$ & 0.74 \\
\hline Heptane & $0 \cdot 068$ & 0.068 & - & $3 \cdot 32$ & $0 \cdot 46$ \\
\hline Hexane & $0 \cdot 064$ & 0.064 & - & $3 \cdot 19$ & 0.47 \\
\hline Cyclohexane & $0 \cdot 138$ & 0.138 & - & $6 \cdot 23$ & 0.39 \\
\hline Diethyl ether & $0 \cdot 080$ & 0.080 & - & $4 \cdot 26$ & 0.49 \\
\hline Ethyl acetate & 0.039 & 0.014 & 0.025 & $2 \cdot 31$ & 1.63 \\
\hline THF & 0.047 & 0.017 & 0.030 & 1.97 & $1 \cdot 14$ \\
\hline DCM & 0.086 & 0.024 & 0.062 & $2 \cdot 64$ & 1.07 \\
\hline TBA & 0.035 & 0.003 & 0.032 & $2 \cdot 13$ & $7 \cdot 08$ \\
\hline Hexanol & $0 \cdot 041$ & 0.005 & 0.036 & 1.08 & $2 \cdot 15$ \\
\hline Pentanol & 0.041 & 0.004 & 0.037 & $1 \cdot 12$ & 2.79 \\
\hline IBA & 0.037 & 0.003 & 0.034 & $2 \cdot 47$ & $8 \cdot 19$ \\
\hline Propanol & 0.038 & 0.002 & 0.036 & 1.47 & $7 \cdot 34$ \\
\hline Ethanol & 0.032 & 0.002 & 0.030 & $2 \cdot 34$ & 11.66 \\
\hline Methanol & 0.022 & 0.001 & 0.021 & 0.96 & $9 \cdot 6$ \\
\hline Acetonitrile & 0.028 & 0.002 & 0.026 & $0 \cdot 66$ & $3 \cdot 28$ \\
\hline DMF & $0 \cdot 04$ & 0.004 & 0.036 & 0.43 & 1.07 \\
\hline DMSO & 0.03 & 0.002 & 0.028 & 4.04 & $20 \cdot 16$ \\
\hline Formamide & $0 \cdot 018$ & 0.001 & 0.017 & $0 \cdot 11$ & $1 \cdot 12$ \\
\hline NMF & $0 \cdot 015$ & - & 0.015 & - & - \\
\hline
\end{tabular}

Quantum yields $(\Phi)$ for M6C in different solvents are tabulated. $\Phi_{L E}$ and $\Phi_{C T}$ are the quantum yields for individual parts. $k_{L E}^{r a \mathrm{~d}}$ and $k_{L E}^{n r}$ denote respectively the radiative and nonradiative rates associated with the LE band. Estimated errors for these calculations are within $\pm 10 \%$ about the average for most of the cases

that are used to calculate these quantities from steady state and time resolved data are already described in many places ${ }^{5}$ and therefore we discuss only the results here. The values of the net quantum yield $\left(\phi_{n e t}\right)$, and those of the individual components, $\phi_{L E}$ and $\phi_{C T}$, for M6C in these solvents are comparable to those for P6C. As expected, the radiative rates are smaller and non-radiative rates larger in protic solvents than those in aprotic solvents. As found earlier ${ }^{5}$ for closely related molecules, both absorption and LE transition moments are found to be solvent insensitive and their values are $\sim 4 \mathrm{D}$ and $\sim 1 \mathrm{D}$, respectively.

The reaction time constants (or simply the reaction times) are obtained by fitting either the LE or CT emission intensity decays of M6C in various polar solvents. As already stated, the emission intensity decay of M6C in most of the polar solvents was found to adequately fit with bi-exponential function of time. One such fitting is shown in figure 4 along with residuals in the bottom panel for M6C in tetrahydrofuran. Parameters obtained from fits for various solvents are summarized in table 3. The representative fitting shown in figure 4 together with the 'goodness-of-fit' parameter $\left(\chi^{2}\right)$ in table 3 suggest that the emission decays of M6C in these polar solvents are indeed bi-exponential functions of time.
In order to ensure that the fast time constant in the LE decay is indeed associated with the LE $\rightarrow$ CT conversion reaction of $\mathrm{M} 6 \mathrm{C}$, we have also analysed the CT emission decays and compared the fast time constants ('rise-time') with those from the LE decays. Representative fits to the intensity emission decays of $\mathrm{M} 6 \mathrm{C}$ in ethyl acetate are shown in figure 5. Fit parameters shown in the inset suggest that the CT rise time is quite close to that associated with the fast component of the LE decay. This means that the fast time constant found in the LE decay is essentially the $\mathrm{LE} \rightarrow \mathrm{CT}$ conversion reaction in $\mathrm{M} 6 \mathrm{C}$ in this solvent. Similar level of agreement has been observed in most of the cases (IRF permitting) and thus the fast time constant is regarded as the ICT reaction time constant in $\mathrm{M} 6 \mathrm{C}$.

We next explore the correlations of $-\Delta G_{r}$ and the forward reaction rate constant $\left(k_{f}\right)$ obtained for M6C in various polar solvents with solvent dielectric field factor and compare them with those of P6C. The following formula has been used to calculate $k_{f}$ from the reaction time $\left(\tau_{r x n}\right)$ and equilibrium constant,

$$
k_{f}=\frac{1}{\tau_{r x n}\left(1+K_{e q}^{-1}\right)} .
$$


Table 3. Decay of M6C associated with LE band: fit parameters.

\begin{tabular}{|c|c|c|c|c|c|c|}
\hline Solvent & $a_{1}$ & $a_{2}$ & $\tau_{1}(n s)$ & $\tau_{2}(p s)^{\mathrm{b}}$ & $\chi^{2}$ & $a_{r x n} / a_{d e c}$ \\
\hline PFH & 1 & & $1 \cdot 30$ & & 1.25 & \\
\hline Heptane & 1 & & $2 \cdot 05$ & & $1 \cdot 34$ & \\
\hline Hexane & 1 & & $2 \cdot 01$ & & $1 \cdot 30$ & \\
\hline Cyclohexane & 1 & & $2 \cdot 22$ & & $1 \cdot 42$ & \\
\hline Diethyl ether & $0 \cdot 80$ & $0 \cdot 20$ & $2 \cdot 32$ & 119 & $1 \cdot 15$ & $0 \cdot 25$ \\
\hline Ethyl acetate & $0 \cdot 20$ & $0 \cdot 80$ & $2 \cdot 92$ & $<20(17)$ & $1 \cdot 14$ & 3.93 \\
\hline THF & $0 \cdot 28$ & 0.72 & 3.06 & 23 & $1 \cdot 10$ & $2 \cdot 61$ \\
\hline DCM & $0 \cdot 29$ & 0.71 & 3.07 & $<20(18)$ & $1 \cdot 28$ & $2 \cdot 42$ \\
\hline TBA & 0.04 & 0.96 & $3 \cdot 22$ & 29 & $1 \cdot 17$ & $27 \cdot 57$ \\
\hline Hexanol & $0 \cdot 11$ & $0 \cdot 89$ & $3 \cdot 19$ & 133 & 1.83 & $8 \cdot 26$ \\
\hline Pentanol & 0.08 & $0 \cdot 92$ & $3 \cdot 50$ & 94 & 1.97 & 11.99 \\
\hline IBA & 0.03 & 0.97 & 2.73 & 30 & $1 \cdot 15$ & $28 \cdot 41$ \\
\hline Propanol & 0.03 & $0 \cdot 97$ & $3 \cdot 27$ & 29 & 1.56 & $29 \cdot 30$ \\
\hline Ethanol & 0.02 & 0.98 & $2 \cdot 96$ & $<20(15)$ & $1 \cdot 11$ & $40 \cdot 67$ \\
\hline Methanol & $0 \cdot 04$ & $0 \cdot 96$ & $2 \cdot 56$ & $<20(15)$ & $1 \cdot 47$ & $27 \cdot 57$ \\
\hline Acetonitrile & 0.09 & $0 \cdot 91$ & $3 \cdot 43$ & $<20(14)$ & 1.09 & $10 \cdot 76$ \\
\hline $\mathrm{DMF}^{\mathrm{a}}$ & 0.51 & 0.49 & $3 \cdot 53$ & 34 & 1.60 & 0.96 \\
\hline DMSO & 0.01 & 0.99 & $3 \cdot 18$ & $<20(5)$ & $1 \cdot 36$ & $70 \cdot 43$ \\
\hline
\end{tabular}

${ }^{a}$ LE emission could not be collected because of its negligible population in this solvent. Note that short time constants of the bi-exponential fit of LE population decays, $\tau_{2}(p s)$ in the above table, are the reaction times in the corresponding solvents. ${ }^{b}$ Numbers in parenthesis in this column denote the values of reaction time constant obtained from fits to the collected emission intensity decays. Since these numbers are smaller than the one-fifth of the instrument response function $(\sim 100 \mathrm{ps})$, it is better to consider them as reaction time-scales faster than 20 ps.

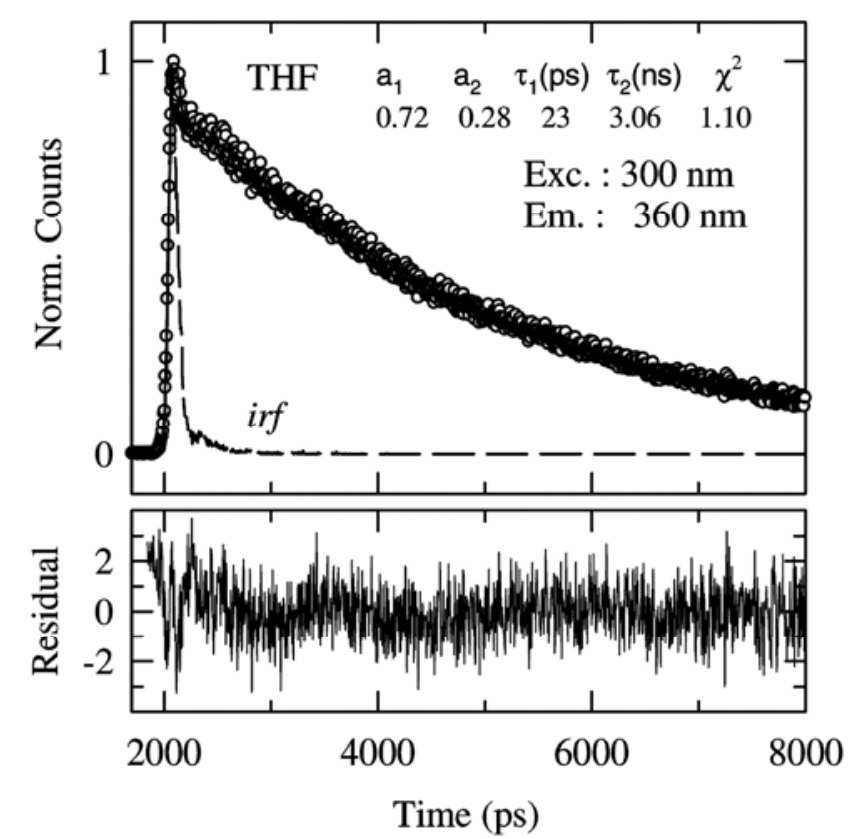

Figure 4. Representative LE emission decay of M6C in tetrahydrofuran (THF, $\varepsilon_{0} \approx 7 \cdot 6$ ) and its bi-exponential fit. The data are represented by the circles, while the fit through the data are by the solid line. The instrument response function is shown by the broken line. The fit (biexponential) results are also provided in the inset. The LE peak count is $\sim 2000$. Residuals are shown in the bottom.
The calculated values of $-\Delta G_{r}\left(=R T \ln K_{e q}\right)$ and $k_{f}$ are shown as a function of $d_{c}\left(\varepsilon_{0}\right)$ with $c=0.25$ in figure 6. The relevant results for $\mathrm{P} 6 \mathrm{C}$ are also shown ${ }^{5}$ (filled circles) in the same figure. A comparison between these two sets of data indicates that the solvent polarity dependence of the reaction driving force $\left(-\Delta G_{r}\right)$ and the reaction rate constant are very similar for these two reactants. This means that the presence of the oxygen atom in the donor moiety does not affect the reaction except decreasing the ground and excited state dipole moments of the reactant.

We next estimate the reaction barrier $\left(\Delta G^{\#}\right)$ that the $\mathrm{LE} \rightarrow \mathrm{CT}$ conversion reaction in $\mathrm{M} 6 \mathrm{C}$ experiences in different polar solvents. In a Kramer's type model, the barrier crossing ${ }^{18-20}$ rate can be expressed

$$
k_{f}=\kappa v_{R} \exp \left(-\Delta G^{\#} / k_{B} T\right),
$$

where $v_{R}$ denotes the reactant well frequency and $\kappa$ the frictional transmission co-efficient $(\kappa \leq 1)$. Solvent dependent $\Delta G^{\#}$ can then be easily estimated from the experimentally obtained $k_{f}$ if we assume that the reactant well frequency for $\mathrm{M} 6 \mathrm{C}$ is the same as that found for P6C $\left(v_{R} \sim 2 \times 10^{12} \mathrm{~s}^{-1}\right)$ and the solvent dynamic effects on the reaction are completely 
absent $(\kappa=1)$. The average of the barrier heights so obtained is found to be approximately $4 k_{B} T$ which is again very close to what has been estimated earlier for $\mathrm{P} 6 \mathrm{C}$ in the same solvent friction limit. ${ }^{5}$

Next we explore how the forward reaction rate constant and the activation energy are correlated to the reaction driving force, $\Delta G_{r}$. Data in figure 7 reveal that a strong correlation between $k_{f}$ and $\Delta G_{r}$ exists (upper panel). Interestingly, the correlation curves indicate different slopes for polar protic and aprotic solvents. Note that similar difference between these two types of solvents has also been observed for the solvent dependence of area ratio and equilibrium constant (see figure 3). Despite the difference in slopes, the strong correlation suggests

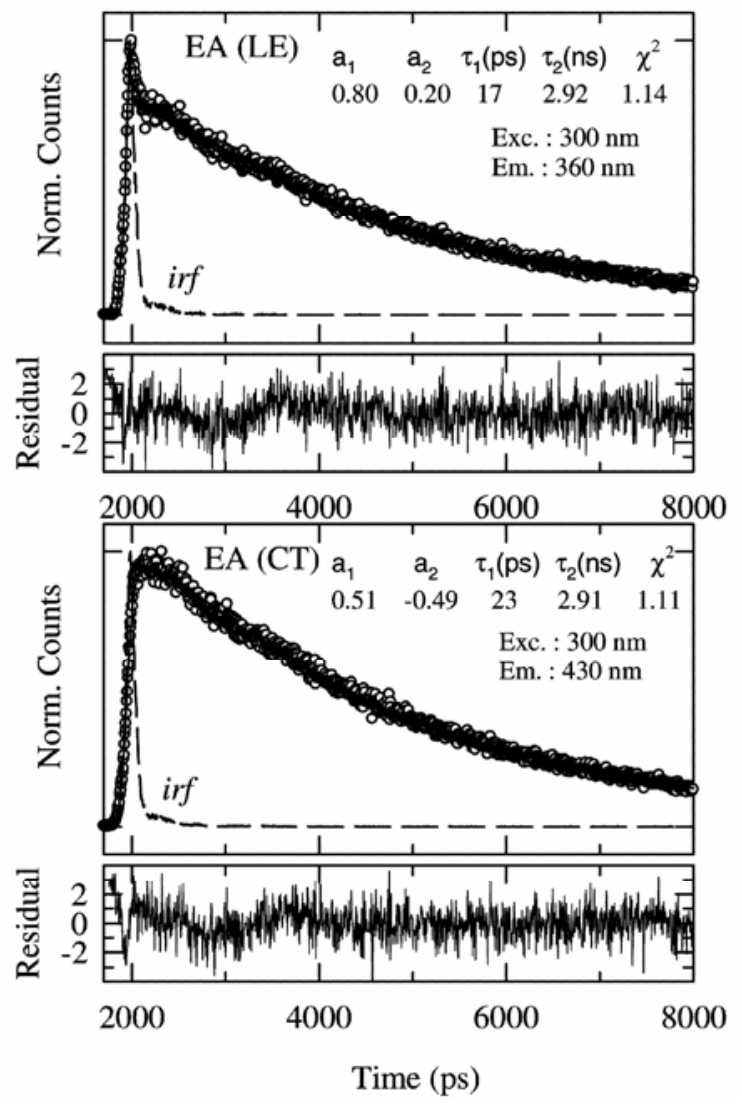

Figure 5. LE emission decay (upper panel) and CT emission decay (lower panel) of M6C in ethyl acetate $\left(\mathrm{EA}, \varepsilon_{0} \approx 6\right)$ and its bi-exponential fit. The line represents a bi-exponential fit through the experimental data (circles). Fit parameters are shown in the inset. Residuals are shown at the bottom of each panel. Fit parameters shown in the inset suggest that the CT rise time is quite close to that associated with the fast component of the LE decay in the upper panel. This means that the fast time constant found in the LE decay is essentially the LE $\rightarrow$ CT conversion reaction in $\mathrm{M} 6 \mathrm{C}$ in this solvent. that the reaction rate constant is significantly influenced by $\Delta G_{r}$ in both types of solvents and this influence arises mainly from the modulation of the barrier height $\left(\Delta G^{\#}\right)$. As shown in the lower panel of figure 7, $\Delta G^{\#}$ is linearly related to $\Delta G_{r}$ for $\mathrm{M} 6 \mathrm{C}$ in these solvents and asserts the relation, ${ }^{5} \Delta G^{\#} \approx \alpha \Delta G_{r}$. Separate linear fits of the data shown in this panel to the above relation produce 0.6 and 0.18 for $\alpha$ in protic and aprotic polar solvents, respectively. The average of these two values is $\sim 0.4$ which is again very close to what has been found earlier for P6C.

An interesting consequence of the different slopes of $\Delta G^{\#}$ versus $\Delta G_{r}$ curves for protic and aprotic polar solvents would be that the reaction in methanol should proceed with a faster rate constant than that in acetonitrile. However, the present experimental data do not show such a difference (see table 3).

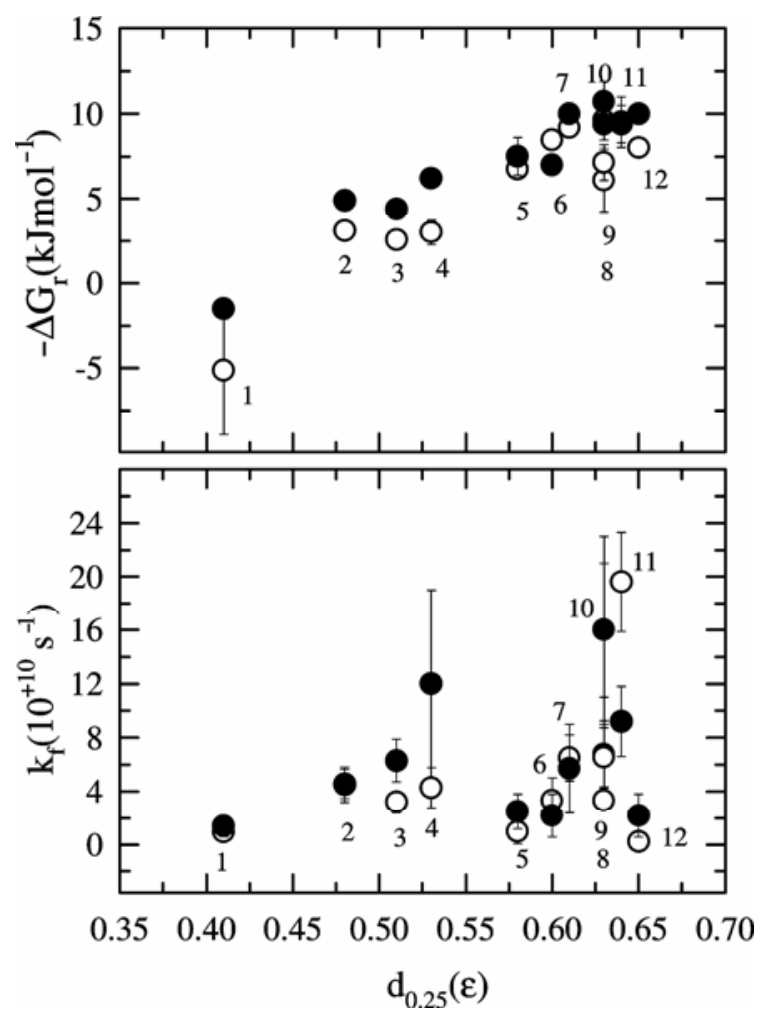

Figure 6. Comparison between $\mathrm{M} 6 \mathrm{C}$ and $\mathrm{P} 6 \mathrm{C}$. Gibbs free energy change $\left(-\Delta G_{r}\right)$ and forward reaction rate constant $\left(k_{f}\right)$ are shown as a function of solvent dielectric field factor. Open and filled symbols represent the data respectively for M6C and P6C. Solvents are marked with numbers, which are assigned as follows: 1 (DEE), 2 (EA), 3 (THF), 4 (DCM), 5 (pentanol), 6 (propanol), 7 (ethanol), 8 (DMF), 9 (acetonitrile), 10 (methanol), 11 (DMSO), 12 (formamide). Note large error bars in P6C data (from ref. 5) for some solvents in which LE area was found to be very small. 
This is because the reaction time constants of M6C in these two 'ultrafast' solvents are much shorter than the effective time resolution of the instrument $(\sim 100 \mathrm{ps})$. The similarity in reaction time constants for P6C in methanol and acetonitrile (7 ps and $8 \mathrm{ps}$ ) was also found earlier with a much better time resolution $(\sim 25 \mathrm{ps}) .^{5}$ Therefore, the present results for M6C in these two solvents ( $\sim 15 \mathrm{ps})$ are in corroboration with what has already been observed earlier with $\mathrm{P} 6 \mathrm{C}$.

The reliability of the measured average reaction times of less than $100 \mathrm{ps}$ which the present study reports (see table 3) for M6C in a number of solvents considered here warrants further discussion. Since measured reaction time in many of the solvents considered here is much faster than the time resolution, any inaccuracy in measurements can give rise to

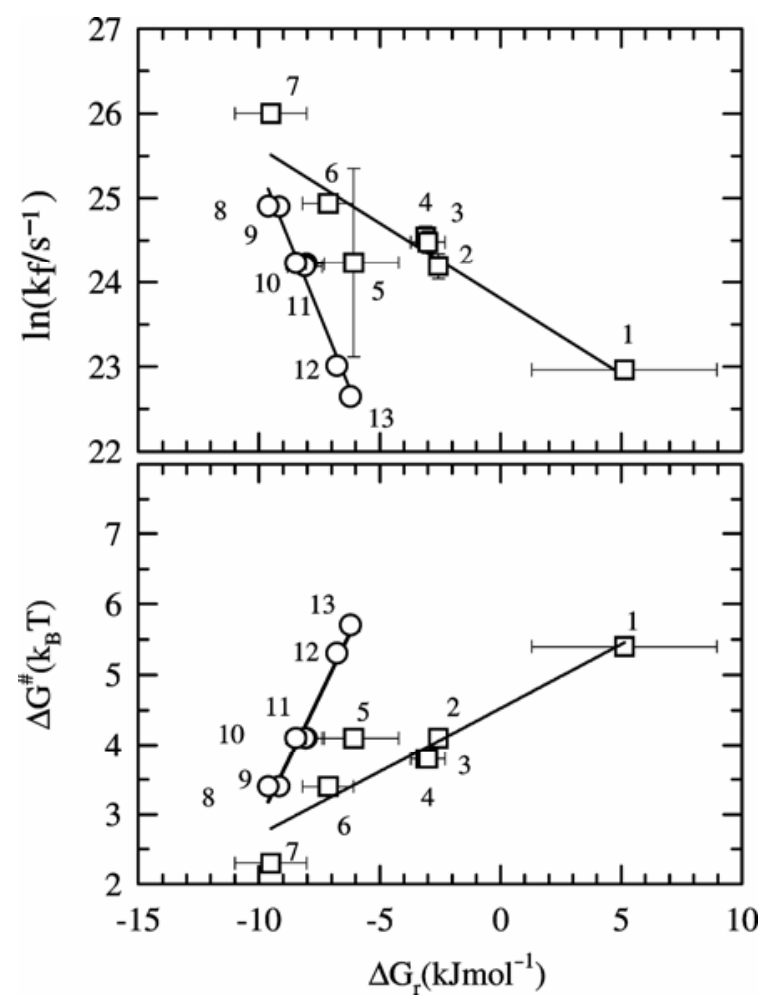

Figure 7. Upper panel: Logarithmic forward rate constant $\left(\ln k_{f}\right)$ versus the reaction driving force $\left(\Delta G_{r}\right)$ of the $\mathrm{LE} \rightarrow \mathrm{CT}$ reaction. Open and filled symbols represent respectively polar protic and aprotic solvents. Solid lines represent linear fits through the data. Solvents are marked with numbers, which are assigned as follows: 1 (DEE), 2 (THF), 3 (DCM), 4 (EA), 5 (DMF), 6 (acetonitrile), 7 (DMSO), 8 (methanol), 9 (ethanol), 10 (propanol), 11 (2methyl-1-propanol), 12 (pentanol), 13 (hexanol). Lower panel: Relationship between the barrier heights $\Delta G^{\#}$ and reaction driving force $\left(\Delta G_{r}\right)$. Representations remain the same as in the upper panel. incorrect values of thermodynamic and kinetic parameters calculated using these reaction times, leading to erroneous conclusions. Fortunately, it has been shown elsewhere that a dynamical event with time constant of as fast as one-fifth of the IRF can be measured with some confidence if iterative reconvolution method is employed to extract fitparameters from the collected intensity decays. ${ }^{5}$ In one of our earlier studies on solute rotation in alcohol solvents, ${ }^{21}$ a comparative study has shown that we could reliably measure a time constant of $\sim 15 \mathrm{ps}$ employing a time resolution of $\sim 75 \mathrm{ps}$ (see table 3 of ref. 21). We expect that this accuracy is also extended to the present measurements and thus reaction times $\sim 20 \geq \tau_{\text {rxn }}(p s) \leq 50$ are semi-quantitatively correct. In addition, our earlier works with TICT reaction of closely related molecules in various media ${ }^{11,13,16(b), 22}$ have reported time constants $<100$ ps measured with time resolution as broad as $\sim 475$ ps. Even though such data may question the reliability of the measurements, our measured time constants with IRF $\sim 475$ ps seemed to have compared well with some previously measured reaction time constants ${ }^{5}$ with time resolution as sharp as $\sim 25 \mathrm{ps}$. These favourable comparisons have led us to believe that the reaction time constants reported here for $\mathrm{M} 6 \mathrm{C}$ in bulk polar solvents are indeed meaningful and can generate a semi-quantitative description of solvent dependence of the TICT reaction in M6C. However, we caution that a time constant of $\sim 5 \mathrm{ps}$ (for DMSO) may not represent the real time scale and thus should be considered with lesser degree of confidence. ${ }^{23}$ In spite of the above discussion, conclusions drawn from this work remain unaltered as the calculation of average equilibrium constant $\left(K_{e q}\right)$ involves only the amplitudes of the decay components, not the reaction time constants.

\section{Conclusion}

To summarize, the replacement of the carbon atom para to the nitrogen atom of the six-membered donor moiety of P6C does not lead to any appreciable change in reaction driving force or activation barrier. Reaction driving force and activation free energy are found to be linearly correlated with an average slope of $\sim 0.4$. The correlation between the reaction rate constant and the estimated activation barrier in the limit of zero solvent friction produces an activation barrier of $\sim 4 k_{B} T$ for this ICT molecule. All these results are similar to those obtained 
for P6C. The only effect of the oxygen replacement in the donor moiety has been the blue shift of the absorption and emission spectra of M6C in a given solvent relative to those of P6C.

Temperature-dependent studies of the reaction rate in these solvents should be carried out to estimate the 'actual' barrier height involved in the ICT reaction of $\mathrm{M} 6 \mathrm{C}$ in these solvents. One may also like to carry out quantum chemical calculations in order to find out why insertion of the oxygen atom induces blue shift in spectra relative to those in P6C. If the difference in slopes between protic and aprotic solvents are any indication of different solutesolvent interactions for M6C, then this molecule's better solubility can be used to study electrolyte effects in water-rich environments. The fast reaction time constant of M6C can also be used to probe the spatial and temporal scales of heterogeneity in molten non-aqueous electrolyte mixtures where the system is expected to behave as super-cooled solutions. ${ }^{24}$ Some of the above works are already in progress and we hope to report them soon.

\section{Acknowledgements}

Financial supports from the Council of Scientific and Industrial Research (CSIR), India is gratefully acknowledged. TP acknowledges the University Grants Commission (UGC), India and HG thanks SN Bose National Centre for Basic Sciences (SNBNCBS) for research fellowships. In addition, HG expresses his sincere thanks to the Vice Chancellor, Aliah University, West Bengal for encouragement.

\section{References}

1. Grabowski Z R, Rotkiewicz K and Rettig W 2003 Chem. Rev. 1033899

2. Lippert E, Rettig W, Bonacic-Koutecky V, Heisel F and Mieche J A 1987 Adv. Chem. Phys. 681
3. Zachariasse $\mathrm{K}$ A, Druzhinin S I, Bosch W and Machinek R 2004 J. Am. Chem. Soc. 1261705

4. Techert S and Zachariasse K A $2004 \mathrm{~J}$. Am. Chem. Soc. 1265593

5. Dahl K, Biswas R, Ito N and Maroncelli M 2005 J. Phys. Chem. B109 1563

6. Rettig W 1986 Angrew. Chem. Int. Ed. 250971

7. Rettig W 1991 Nachr. Chem. Tech. Lab. 39298

8. Habib Jiwan J L and Soumillion J P 1992 J. Photochem. Photobiol. A64 145

9. Rettig W and Wolfbesis O S 1993 Fluorescence spectroscopy new methods and applications (Springer Berlin) p. 31

10. Plaza P, Jung N D, Martin M M, Meyer Y H, Vogel $\mathrm{M}$ and Rettig W 1992 Chem. Phys. 1680365

11. Pradhan T, Ghoshal P and Biswas R 2008 J. Phys. Chem. A112 915

12. Pradhan T and Biswas R 2007 J. Phys. Chem. A111 11514

13. Pradhan T and Biswas R 2007 J. Phys. Chem. A111 11524

14. Pradhan T, Ghoshal P and Biswas R 2009 J. Chem. Sci. 12195

15. Braun D and Rettig W 1997 Chem. Phys. Lett. 268 110

16. (a) Biswas R, Rohman N, Pradhan $T$ and Buchner R 2008 J. Phys. Chem. B112 9379; (b) Pradhan T, Gazi H A R and Biswas R 2009 J. Chem. Phys. 131 054507

17. (a) Rettig W 1980 J. Luminesc. 26 21; (b) Rettig W 1982 J. Phys. Chem. 861970

18. Zwan vander G and Hynes J T 1991 Chem. Phys. 152 169

19. Hynes J T 1985 The theory of reactions in solution in the theory of chemical reactions (ed.) M Baer and I G Crizmadia (Boca Raton: CRC Press) p. 171

20. Fleming G R and Hanggi P 1993 Activated barrier crossing (Singapore: World Scientific)

21. Pradhan T, Ghoshal P and Biswas R $2008 \mathrm{~J}$. Chem. Sci. 120275

22. Pradhan T and Biswas R 2009 J. Soln. Chem. 38 517

23. Bevington P R 1969 Data reduction and error analyses for the physical sciences (New York: MacGrawHill)

24. Guchhait B, Gazi H A R, Kashyap H K and Biswas R 2010 J. Phys. Chem. B114 5066 